\title{
Efecto de sustratos orgánicos en plantas de fresa (Fragaria sp.) cv 'Albion' bajo condiciones de campo
}

\section{Organic substrates effect in strawberry $\mathrm{Cv}$ 'Albion' (Fragaria sp.) plants, under field conditions}

Fecha de recepción: 21 de marzo de 2016

Fecha de aceptación: 1 de junio de 2016

José Samuel Medina-Bolívar ${ }^{1}$

Elberth Hernando Pinzón-Sandoval ${ }^{2}$

German Eduardo Cely ${ }^{3}$

\section{Resumen}

El cultivo de fresa es una de las opciones productivas para los agricultores, principalmente de los departamentos de Cundinamarca, Antioquia y Boyacá. La tendencia creciente hacia una agricultura cada vez más abierta al comercio mundial determina que las actividades agrícolas nacionales deben ser cada día más productivas y competitivas, esto soportado en nuevas y mejores tecnologías de producción. Dentro de estas, se cuenta el uso de diferentes sustratos con el fin de solucionar problemas como acidez, alta erosión o problemas de tipo fitosanitario que impiden la siembra de forma directa en el suelo. Teniendo en cuenta lo anterior el objetivo de la investigación fue la evaluación de diferentes sustratos orgánicos en el crecimiento y desarrollo de plantas de fresa cv 'Albion', bajo condiciones de campo abierto. Para la evaluación se utilizó un diseño completamente al azar con tres tratamientos siendo S1: suelo 50\% + fibra de coco 50\% (S:FC), S2: suelo 50\% + fibra de coco 25\% + cascarilla de arroz 25\% (S:FC:CA) y S3: suelo $50 \%$ + cascarilla de arroz 50\% (S:CA), cada uno conto con tres replicaciones. Las variables evaluadas fueron masa fresca y seca de raíz y parte aérea, área foliar, clorofilas totales, numero de frutos/planta y producción g/planta. El tratamiento (S1), mostró diferencias significativas según la prueba de Tukey $(\mathrm{P} \leq 0.05)$ en la mayoría de parámetros evaluados, esto lo convierte en una alternativa para la producción de fresa cv 'Albión' bajo las condiciones de estudio.

Palabras claves: frutilla; hidroponía; materiales orgánicos; medio de crecimiento.

1 Profesional Independiente. Estudiante Maestría en Ciencias Agrarias.

2 M.Sc. Universidad Pedagógica y Tecnológica de Colombia (Tunja-Boyacá, Colombia).

3 M.Sc. Universidad Pedagógica y Tecnológica de Colombia (Tunja-Boyacá, Colombia).german.cely@uptc.edu.co. 


\begin{abstract}
The strawberry crop is one of the production options for farmers, mainly in the departments of Cundinamarca, Antioquia and Boyacá. The increasingly open world trade growing trend towards agriculture determines that national agricultural activities should be more productive and competitive every day, it supported these new and improved production technologies. Within these substrates using different order to solve problems like acidity, high erosion or phytosanitary problems that prevent direct sowing in the soil is counted. Given the above, the objective of the research was the evaluation of different organic substrates in the growth and development of strawberry plants cv 'Albion', under field conditions. was used for evaluation as a completely randomized design with three treatments being S1: soil 50\% + coir $50 \%$ (S: FC), S2: soil 50\% + rice husk 25\% + coir 25\% (S: FC: CA) and S3: soil 50\% + 50\% rice hull (S: $\mathrm{CA}$ ), each with three replications conto. The variables evaluated were fresh and dry root mass and aerial part, leaf area, total chlorophylls, number of fruits/plant and production g/plant. Treatment (S1), showed significant differences according to Tukey test $(P \leq 0.05)$ in most parameters assessed, this makes for an alternative for the production of strawberry cv 'Albion' under the study conditions.
\end{abstract}

Keywords: growth medium; hydroponics; organic materials; strawberry. 


\section{Introducción}

El cultivo de fresa es una de las opciones productivas para los agricultores, principalmente de los departamentos de Cundinamarca, Antioquia y Boyacá en los que se concentra el $88.8 \%$ de la producción nacional (1). La fresa es una fruta apreciada a nivel mundial por su aroma, color y textura (2). Es fuente importante de compuestos bioactivos como vitamina $\mathrm{C}$, K, filoquinona, folato y constituyentes fenólicos con capacidad antioxidante, siendo consumida tanto en fresco como procesada (3). Dentro de los cultivares utilizados para la siembra en Boyacá se destaca el cv 'Albión' debido a que presenta características como frutos de excepcional calidad, representada en su tamaño, sabor y firmeza; un peso promedio de 32 g/fruto; fácil recolección y excelente vida en anaquel (4). Esto hace que el cultivar sean muy apetecido por los productores y consumidores.

La tendencia creciente hacia una agricultura cada vez más abierta al comercio mundial determina que las actividades agrícolas nacionales deben ser cada día más eficientes y competitivas, esto soportado en nuevas y mejores tecnologías de producción (5), donde se destaca el uso de diferentes sustratos con el fin de solucionar problemas como: acidez, alta erosión o problemas de tipo fitosanitario que impiden la siembra de forma directa en el suelo. Además de lo anterior las zonas de producción cada vez son menores lo que implica que se tenga que hacer uso de zonas que antes eran marginales siendo fundamental el uso de sustratos. El término sustrato, se aplica en horticultura a todo material sólido distinto del suelo natural o de síntesis, mineral u orgánico, que puesto en un contenedor en forma pura o en mezcla, permite el anclaje del sistema de raíces (6). Por otra parte, debe señalarse que el cultivo de plantas en sustrato, permite un control del medio ambiente radical, particularmente en aspectos relacionados con el suministro de agua y nutrientes (7).

Los sustratos empleados pueden diferenciarse como orgánicos, por ejemplo, tierra, turba, compost, fibra de coco o cascarilla de arroz e inorgánicos como perlita, vermiculita o arena (8). Den- tro de los sustratos orgánicos más utilizados se encuentra la cascarilla de arroz debido a que es un material de fácil adquisición y bajo costo (6). Este presenta una baja tasa de descomposición por su alto contenido de sílice, pero que puede ser mejorado mediante quema parcial o tostión; dentro de sus características físicas presenta elevada porosidad y aireación, además, alta capacidad de retención de agua después del proceso de quema parcial (9). La fibra de coco es un sustrato orgánico de lenta descomposición debido a su contenido de lignina que puede ser de hasta un $45 \%$; se obtiene del mesocarpo del fruto de cocotero, mediante un proceso de trituración y cribado $(10,11)$. Poseen una excelente retención de humedad ya que al rehidratarse puede aumentar su volumen hasta 3,5 veces el volumen inicial y puede tener una vida útil de hasta 6 años (9). Las propiedades físicas de este sustrato muestran una similitud con las características de las turbas rubias en cuanto al tamaño de partícula, inercia térmica, retención de humedad, aireación y drenaje $(10,11)$. Este material se conocía y utilizaba desde tiempo inmemorial por los agricultores de las zonas tropicales (12).

Las características físicas de los sustratos, o medios de crecimiento, son cruciales para su uso efectivo y en gran medida condiciona el potencial productivo del cultivo, pues constituyen el medio en el que se desarrollarán las raíces, las cuales tienen gran influencia en el crecimiento y desarrollo de las plantas (13). Un buen sustrato debe estar acorde con las exigencias de nutrientes, agua y aire de la especie a propagar y así garantizar un buen soporte, suministrar humedad y aireación adecuadas, presentar bajo costo, ser de fácil adquisición y que no libere sustancias que puedan afectar el establecimiento y crecimiento (14). Normalmente, es difícil que un solo material reúna las características ideales para el desarrollo de los cultivos (15). Por lo anterior la combinación de sustratos puede lograr un efecto conjunto de las mejores características de estos, y así buscar mejores condiciones para el crecimiento y desarrollo de la especie cultivada.

A pesar de que en Colombia el uso de sustratos se ha ido posesionando en muchos sistemas de 
producción hortofrutícolas, las investigaciones sobre el empleo de los mismos son escasas (16). Por lo anterior el objetivo de la investigación fue la evaluación de diferentes sustratos orgánicos en el crecimiento y desarrollo de plantas de fresa cV 'Albion', bajo condiciones de campo abierto en el municipio de Tunja-Boyacá.

\section{Materiales y métodos}

El ensayo se estableció en la finca San Carlos, vereda Germania, municipio de Tunja-Boyacá, con coordenadas $5^{\circ} 31^{\prime} 23^{\prime \prime} \mathrm{N}$ y $73^{\circ} 24^{\prime} 12^{\prime \prime} \mathrm{O}$, una altura de $2.680 \mathrm{msnm}$, precipitación anual promedio de $900 \mathrm{~mm}$, temperatura promedio de $13^{\circ} \mathrm{C}$ y humedad relativa del $80 \%$.

El material vegetal que se utilizó fueron esquejes certificados por Proplantas ${ }^{\circledR}$ del cultivar 'Albión' los cuales provienen de proceso de estratificación en cuarto frio a una temperatura de $-1^{\circ} \mathrm{C}$ y humedad relativa del $90 \%$ durante un periodo de
6 meses, estos fueron aclimatados a temperatura ambiente de $14{ }^{\circ} \mathrm{C}, 24 \mathrm{~h}$ antes de la siembra.

Se utilizó un diseño completamente al azar, con 3 tratamiento cada uno, con 3 repeticiones para un total de 9 unidades experimentales, cada una compuesta por una cama construida con una canal de $12 \mathrm{~m}$ de longitud de forma rectangular con base de $75 \mathrm{~cm}$ y altura de $30 \mathrm{~cm}$ con cobertura negro plata de calibre $1.5 \mathrm{~mm}$. Las plantas fueron sembradas a una distancia de $25 \mathrm{~cm}$, se instaló una línea de riego con goteros auto compensados a 15 $\mathrm{cm}$ y un caudal de 1.2 L/hora, esto en cada una de las camas. Los tratamientos fueron los siguientes: S1: suelo $50 \%$ + fibra de coco $50 \%$ (S:FC); S2: suelo $50 \%+$ fibra de coco $25 \%+$ cascarilla de arroz $25 \%$ (S:FC:CA) y S3: suelo $50 \%+$ cascarilla de arroz 50\% (S:CA). La cascarilla de arroz fue previamente quemada buscando mejorar algunas de sus propiedades. Todos los materiales utilizados como sustratos fueron desinfectados con una solución de hipoclorito $0.1 \%$. Las Propiedades físicas de los sustratos se presentan en la Tabla I.

Tabla I. Propiedades físicas de los sustratos usados en el crecimiento y desarrollo de plantas de fresa cv 'Albión', a campo abierto en Tunja-Boyacá. *(17); ** (18)

\begin{tabular}{|c|c|c|c|c|}
\hline & \multicolumn{4}{|c|}{ Propiedades Físicas } \\
\hline Sustrato & PT (\%) & PA (\%) & RH (\%) & Da $\left(\mathrm{g} \mathrm{cm}^{-3}\right)$ \\
\hline Ideal* $^{*}$ & $70-85$ & $10-20$ & $55-70$ & $<0.4$ \\
Fibra de coco** & 81.8 & 16.3 & 65.5 & 0.07 \\
Cascarilla de arroz $^{* *}$ & 84.8 & 62.2 & 16.6 & 0.09 \\
\hline
\end{tabular}

PT: Porosidad total; PA: Porosidad de aireación; RH: Retención de humedad; Da: densidad aparente.

Las variables de respuesta medidas al cabo de 210 días después de la siembra (dds) fueron: masa fresca de raíz y parte aérea mediante balanza electrónica Acculab VIC 612 de $0.01 \mathrm{~g}$ de precisión; masa seca de raíz y parte aérea, se secaron las muestras en estufa de secado Memmert a $85^{\circ} \mathrm{C}$ hasta que se alcanzó peso constante; área foliar usando un medidor portátil modelo $\mathrm{Cl}-202$ Seedmech; contenido de clorofilas totales mediante clorofilómetro Minolta Spad 502 plus, se realizaron 10 mediciones por planta, de esta forma al final de cada medición se generó un promedio de clorofilas totales por UE, expresada en unidades SPAD; Numero de frutos y producción por planta.

Los datos obtenidos fueron sometidos a pruebas de normalidad y homogeneidad de varianza mediante las pruebas de Shapiro-Wilk y Levene respectivamente, comprobados los supuestos se realizó análisis de varianza, las variables que mostraron diferencias estadísticas, fueron sometidas a pruebas de comparación de medias de Tukey $(\mathrm{P} \leq 0.05)$, los análisis se realizaron con el programa estadístico SAS v.9.2e (SAS Institute Inc., Cary, NC). 


\section{Resultados y discusión}

\section{A. Masa fresca}

Se presentaron diferencias significativas $(P \leq 0.05)$ entre tratamientos para las variables peso fresco de raíz y parte aérea. El peso fresco de la raíz presentó el mayor valor con $263.54 \pm 3.5$ g correspondiente al tratamiento S:FC:CA (S2), el menor valor fue de $72.71 \pm 8.7 \mathrm{~g}$, el cual se obtuvo con el tratamiento S:CA (S3). Por su parte para el peso fresco de la parte aérea el tratamiento S:FC (S1) presentó el mayor valor con $273.03 \pm 1.5 \mathrm{~g}$, mientras que el tratamiento S:CA mostro el valor mínimo con $195.54 \pm 6.4 \mathrm{~g}$ (Figura 1A y 1B).
La masa fresca de la raíz presentó una mayor acumulación al generar una combinación de materiales orgánicos e inorgánicos, debido a que la cascarilla de arroz presenta una alta porosidad de aireación evitando la compactación del sustrato, la fibra de coco aporta una alta retención de humedad y el suelo suministra nutrientes y da estabilidad al sustrato. Lo anterior se refleja en el buen desarrollo de la masa radical por parte de las plantas de fresa con la aplicación del tratamiento S:FC:CA. En plantas de fresa cv 'Oso grande' y 'Chandler', al usar fibra de coco en un $25 \%$ del total del sustrato se obtuvieron los mayores valores para las variables peso fresco y seco de raíz (19). Esto concuerdan con lo observado en el presente estudio (Figura 1A y 2A).

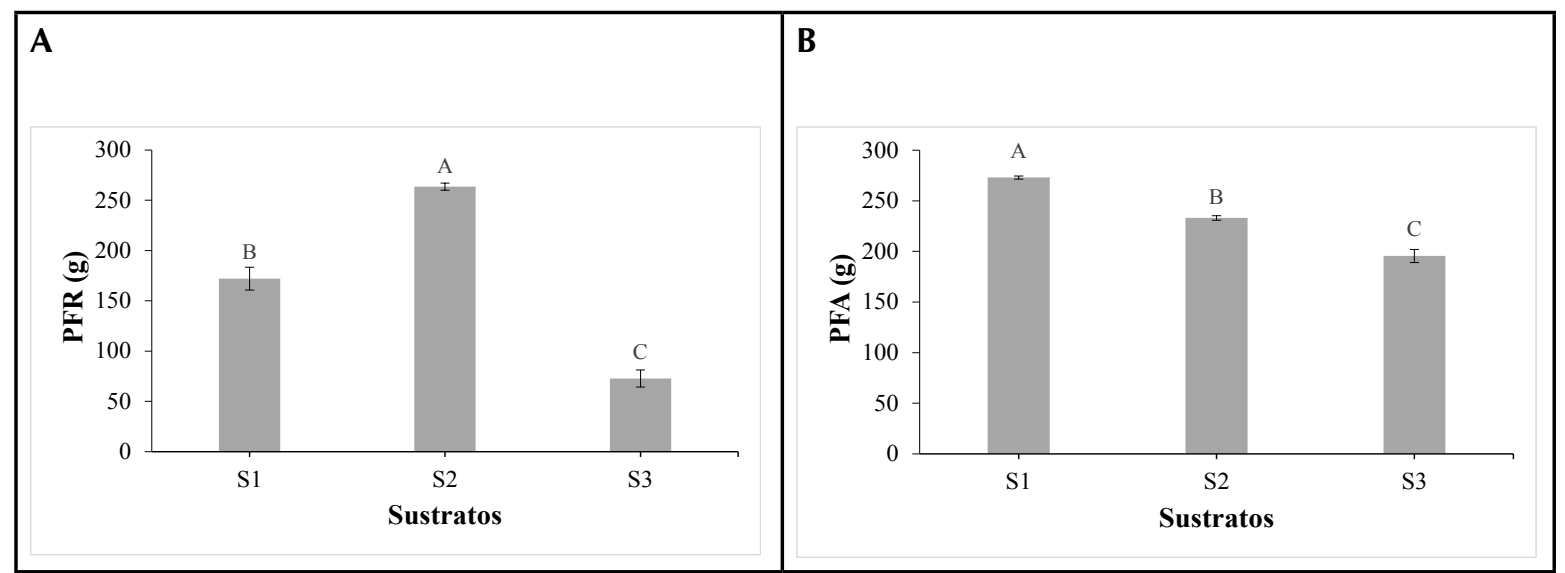

Fig. 1. Comportamiento del peso fresco en planta de fresa cv 'Albión' cultivadas en diferentes sustratos. S1: suelo $50 \%$ + fibra de coco 50\%; S2: suelo 50\% + fibra de coco $25 \%$ + cascarilla de arroz $25 \%$; S3: suelo 50\% + cascarilla de arroz 50\%. A. Peso fresco de raiz (PFR); B. Peso fresco de parte aérea (PFA). Medias con letras distintas presentas diferencias significativas según la prueba de Tukey $(\mathrm{P} \leq 0.05)$, barras verticales corresponden al error estándar $(n=3)$.

El comportamiento de la acumulación de masa fresca en la parte aérea (hojas y tallos), no presentó el mismo comportamiento que se observó en la acumulación de masa fresca de raíz, posiblemente debido al incrementó en la proporción de fibra de coco, que aumentó la retención de humedad disminuyendo la aireación en la rizósfera, esto ocasionó una disminución del crecimiento radical por procesos anaeróbicos (19). Sin embargo al ser un sistema que cuenta con riego y fertilización localizado (riego por goteo), la raíz se puede ubicar en la parte superior del sustrato con lo que reduce su volumen, pero no se interfiere con la toma de agua y nutrientes necesarios para generar un crecimiento aéreo adecuado como se puedo observar (Figura 1B y 2B). $\mathrm{Al}$ estudiar diferentes mezclas de sustratos orgánicos e inorgánicos en el crecimiento del plantas de fresa cv 'Camarosa', se observó que las propiedades físicas como la aireación, porosidad y retención de humedad del sustrato están estrechamente ligadas al desarrollo vegetativo (19). Como se evidencio en la presente investigación. 


\section{B. Masa seca}

La variable presentó diferencias significativas entre tratamientos $(\mathrm{P} \leq 0,05)$, el tratamiento S:FC:CA mostró el mayor valor con $124.7 \pm 3.0 \mathrm{~g}$, mientras que el tratamiento $\mathrm{S}: \mathrm{CA}$ obtuvo el menor valor con $36.73 \pm 1.36 \mathrm{~g}$; esto para el peso seco de raíz. Por su parte el peso seco de parte aérea presentó un valor de $71.83 \pm 1.48 \mathrm{~g}$ para el tratamiento S:FC, y un valor de $49.45 \pm 5.04 \mathrm{~g}$ en el tratamiento $\mathrm{S}: \mathrm{CA}$ (Figura 2A y 2B).

Los valores de materia seca de la raíz están asociados de forma directa con la acumulación de materia fresca de las mismas, esto debido a que la formación de tejidos es la traducción de una adecuada absorción del agua y los nutrientes por parte del sistema radical, en beneficio de la parte aérea de la planta (6); sin embargo esta acumulación también está relacionada con las propiedades de los sustratos ya que algunos pueden presentar un mayor contenido de nutrientes y una mejor retención de humedad (19). Se pudo observar que si bien hubo diferencias estadísticas en cuanto a la acumulación de masa seca de raíz entre los tratamientos S1 y S2, esta no se reflejó en cuanto a la acumulación de masa seca de la parte aérea ya que no se presentaron diferencia estadísticas entre estos dos tratamientos en cuanto a esta variable. Como se mencionó anteriormente al tener riego y fertilización localizados, un sistema radical de poco volumen logro ser suficiente para generar un adecuado crecimiento aéreo de las plantas.

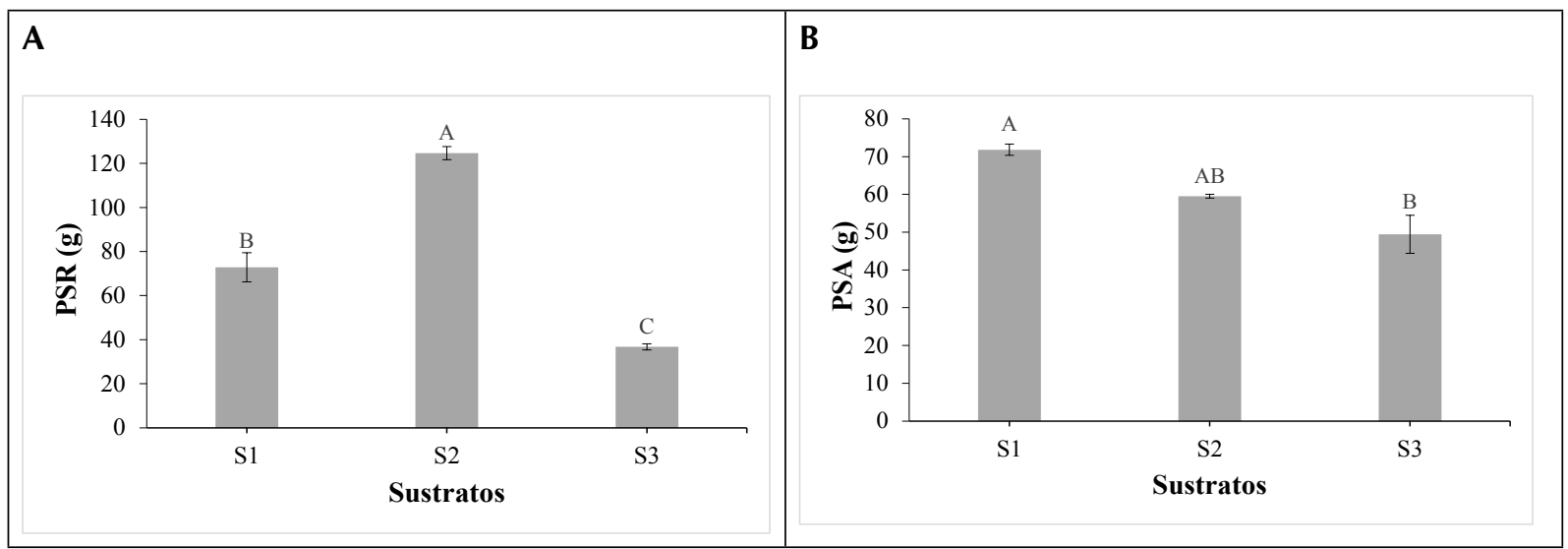

Fig. 2. Comportamiento del peso seco en planta de fresa cv 'Albión' cultivadas en diferentes sustratos. S1: suelo $50 \%$ + fibra de coco $50 \%$; S2: suelo $50 \%$ + fibra de coco $25 \%$ + cascarilla de arroz 25\%; S3: suelo $50 \%$ + cascarilla de arroz 50\%. A. Peso fresco de raíz (PSR); B. Peso fresco de parte aérea (PSA). Medias con letras distintas presentas diferencias significativas según la prueba de Tukey $(P \leq 0.05)$, barras verticales corresponden al error estándar $(n=3)$.

Por su parte el tratamiento Suelo $50 \%+$ Cascarilla $50 \%$ (S3), presentó los menores valores en cuanto a la acumulación de masa seca y fresca a nivel de raíz y parte área, esto debido a que la cascarilla de arroz quemada (CA), presenta una baja retención de humedad y una alta porosidad de aireación (Tabla I), esto ocasiona que la planta tenga una menor disponibilidad de agua y nutrientes durante todo su ciclo fenológico.

\section{C. Área foliar}

Se presentaron diferencias significativas entre los tratamientos $(\mathrm{P} \leq 0.05)$, la mezcla de suelo + fibra de coco (S1), mostró un valor de $1482.78 \pm 43.20$ $\mathrm{cm}^{2}$ siendo el mayor valor para esta variable, por su parte el tratamiento suelo + cascarilla de arroz (S3), mostro el menor valor con $1113.83 \pm 27.57$ $\mathrm{cm}^{2}$ (Figura 3A). 


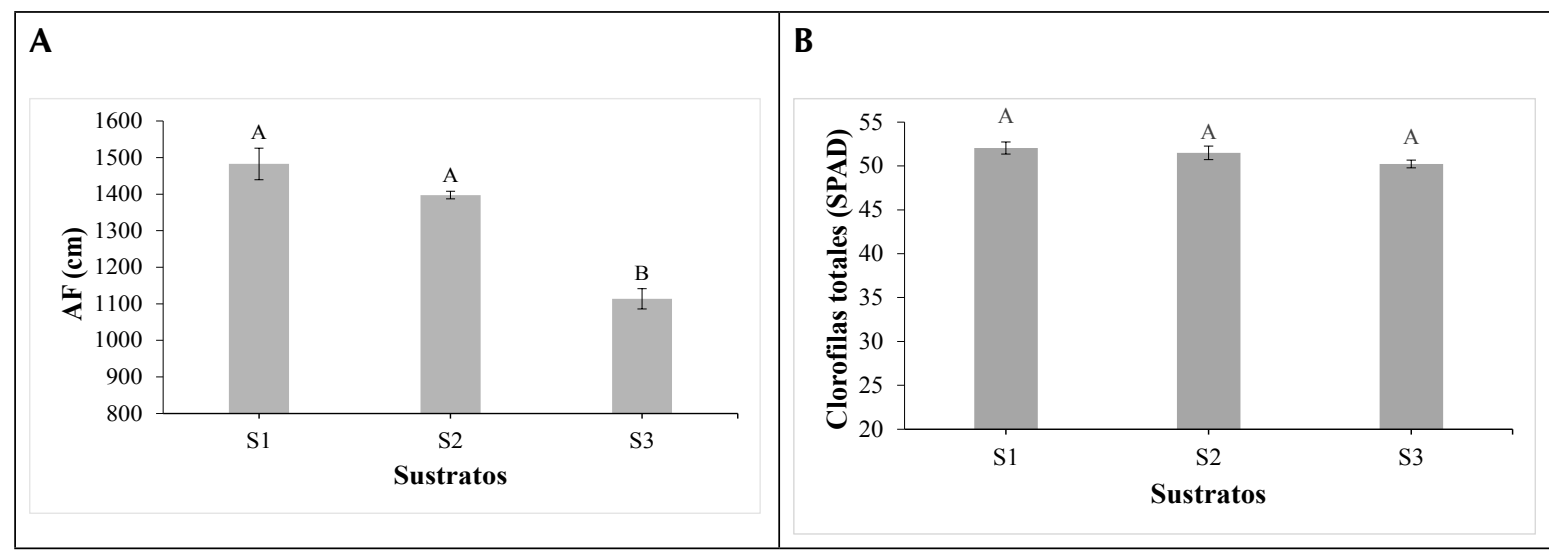

Fig. 3. Comportamiento del área foliar y el contenido de clorofilas en planta de fresa cv 'Albión' cultivadas en diferentes sustratos. S1: suelo $50 \%$ + fibra de coco $50 \%$; S2: suelo $50 \%$ + fibra de coco $25 \%$ + cascarilla de arroz 25\%; S3: suelo 50\% + cascarilla de arroz 50\%. A. Área Foliar (AF); B. Clorofilas totales. Medias con letras distintas presentas diferencias significativas según la prueba de Tukey $(P \leq 0.05)$, barras verticales corresponden al error estándar $(n=3)$.

El área foliar tiene un papel muy importante en el proceso fotosintético, ya que a mayor área foliar, mayor captación de energía lumínica reflejada en mayor cantidad de fotoasimilados (21). Las plantas con mayor área foliar y condiciones favorable, son capaces de aprovechar mejor la energía solar haciendo más eficiente el proceso fotosintético (22). Se observó un aumento significativo del área foliar en los tratamientos que tenían en alguna proporción fibra de coco (S1 y S2), esto concuerda con lo observado en plantas de fresa cv 'Camarosa' cultivadas en vernicompost o aserrín de coco en las que se presentó un aumento significativo en el número de hojas (20). El número de hojas en las variedades 'Oso grande' y 'Chandler' a los 125 días después de establecimiento, aumentó de forma significativa cuando se utilizó como sustrato una mezcla de tezontle y fibra de coco, obteniendo valores de 619.9 y $650.4 \mathrm{~cm}^{2}$ respectivamente (19).

El contenido de clorofilas totales no mostro diferencias estadísticas (Figura 3B). Debido a que el método utilizado fue tipo no destructivo y él cual puede ser utilizado para monitorear el contenido de $\mathrm{N}$ en la hoja $(23,24)$. Se puede inferir entonces que las diferentes mezclas de materiales que conformaron los tratamientos, permitieron una adecuada disponibilidad de nutrientes entre estos el Nitrógeno y el Magnesio que son componentes fundamentales de la molécula de clorofila (25).
Si bien se indican que sustratos asociados con la presencia de cascarilla de arroz y fibra de coco, realizan poco aporte nutricional (26), estos dentro de los sustratos orgánicos son de los mejores en cuanto a las características físicas, facilitando el desarrollo radical y permitiendo una buen disponibilidad de agua y nutrientes por las plantas, siendo una opción cuando se trabajan sistemas de fertiirrigación como se dio en el presente estudio.

\section{Producción}

El número de frutos por planta mostro diferencias estadísticas $(P \leq 0.05)$ entre tratamientos. El tratamiento compuesto por la mezcla de suelo + fibra de coco $(\mathrm{S} 1)$, presentó un promedio de $11.7 \pm 1.2$ frutos/planta siendo este el máximo valor para esta variable, mientras que en el tratamiento de suelo +cascarilla de arroz se observó el menor promedio con $6.0 \pm 0.57$ frutos/planta (Figura 4A). Estos resultados se asocian con lo observado en la variable área foliar (Figura 3A), ya que se ha descrito que un mayor número de hojas o una mayor área foliar está directamente relacionada con una mayor tasa fotosintética, convirtiéndola en la principal fuente de fotoasimilados (azúcares, hormonas, aminoácidos, etc.) y nutrientes, así obtener un mayor movimiento de los mismos hacia los órganos vertedero en este caso los frutos (27). Se ha reportado que la producción de fresa cv 'Camarosa' aumentó hasta 
en un $83 \%$ cuando se utilizó como sustrato aserrín de coco, esto en relación al testigo (20).

La variable producción mostro diferencia estadísticas entre tratamientos $(\mathrm{P} \leq 0.05)$, el tratamiento $\mathrm{S}: \mathrm{FC}$ presentó el mayor valor para esta variable con $70.33 \pm 2.35 \mathrm{~g} /$ planta, mientras el tratamiento $\mathrm{S}: \mathrm{CA}$ mostro el menor valor con $43.88 \pm 2.17 \mathrm{~g} /$ planta (Figura 4B). Lo anterior concuerda con lo observado en miniclavel var. Ronny (Dianthus caryophyIlus L.) en el que al utilizar fibra de coco como sustrato se aumentó la producción en un $9.5 \%$ en comparación al sustrato convencional (28).

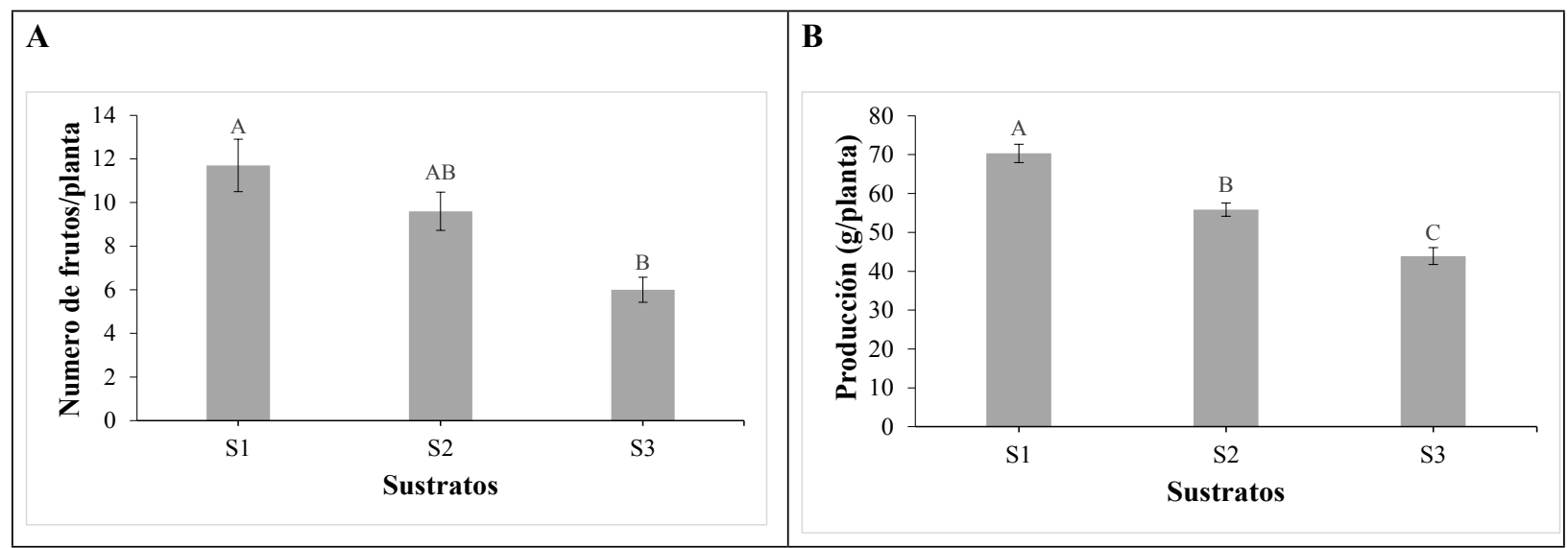

Fig. 4. Comportamiento de planta de fresa cv 'Albión' cultivadas en diferentes sustratos. S1: suelo $50 \%$ + fibra de coco 50\%; S2: suelo 50\% + fibra de coco 25\% + cascarilla de arroz 25\%; S3: suelo $50 \%$ + cascarilla de arroz 50\%. A. Número de frutos; B. Producción. Medias con letras distintas presentas diferencias significativas según la prueba de Tukey $(\mathrm{P} \leq 0.05)$, barras verticales corresponden al error estándar $(n=3)$.

Los resultados encontrados en cuanto al número de fruto/planta y la producción g/planta, pueden explicarse, teniendo en cuenta que al generar un sustrato apropiado para el crecimiento de las plantas, se debe buscar una alta capacidad de retención de agua, suficiente aireación, baja densidad aparente, alta porosidad total y fácil hidratación (15). Esto se logró al realizar mezcla entre un sustrato de tipo inorgánico (fibra de coco) y uno de tipo orgánico (suelo), así combinar las mejores características físicas de cada uno. De esta forma las plantas aprovecharon eficientemente el agua y los nutrientes suministrados de forma localizada, lo cual redunda en mayor producción de frutos (29).

\section{Conclusiones}

El tratamiento compuesto por la mezcla de suelo + fibra de coco (S1) en relación 1:1, mostró los mejores valores para las variables peso fresco y seco de parte aérea, área foliar, numero de frutos/ planta y producción g/planta. Esto lo convierte en una alternativa para la producción de fresa cv 'Albión' bajo las condiciones de estudio.

De los materiales evaluados la fibra de coco fue el que mejor comportamiento mostró, ya que favoreció el crecimiento de plantas de fresa $\mathrm{cv}$ 'Albión' cuando se empleó en diferentes porcentajes, acompañado de sustratos orgánicos e inorgánicos. Mientras que la cascarilla de arroz no presentó un buen comportamiento pues mostró los valores más bajos en todos los parámetros evaluados.

\section{Referencias}

(1) Ministerio de Agricultura y Desarrollo Rural. Anuario estadístico de frutas y hortalizas 2007-2011, (en línea) 2012 (fecha de acceso 20 de enero de 2016); pag. 62. Disponible en: http://www.agronet.gov.co/www/htm3b/ public/Anuario/anuario $\% 20$ estadistico $\% 20$ de $\% 20$ frutas $\% 20 y \% 20$ hortalizas $\% 202011$. pdf. 
(2) Khoshnevisan B, Rafiee S, Mousazedh H. Enviromental impact assessment of open field and greenhouse strawberry production. Eur. J. Agron, 2013; 50: 29-37. DOI: http://dx.doi. org/10.1016/j.eja.2013.05.003.

(3) Giampieri F, Tulipani S, Alvarez-Suarez JM, Quiles JL, Mezzetti B, Battino D. The strawberry: composition, nutritional quality, and impact on human health. Nutrition, 2012; 28(1): 9-19. DOI: http://dx.doi.org/10.1016/j. nut.2011.08.009.

(4) Santoyo J, Martínez, C. Paquete tecnológico para la producción de fresa, (en línea) 2016, (fecha de acceso 20 de febrero de 2016); pag. 10. Disponible en: http://www.fps.org.mx/ divulgacion/attachments/article/814/Paquete $\% 20$ tecnologico $\% 20$ para $\%$ 20la \% 20producci $\%$ C3\%B3n\%20de\%20fresa.pdf.

(5) Ruiz R, Piedrahíta W. Fresa (Fragaria x ananassa). Manual para el cultivo de frutales en el trópico. Produmedios, Bogotá. 2012. pp 474495.

(6) López F, Guío N, Fischer G, Miranda D. Propagación de uchuva (Physalis peruviana L.) mediante diferentes tipos de esquejes y sustratos. Rev.Fac.Nal.Agr.Medellín, 2008; 61(1): 4347-4357.

(7) Cegarra J. Compostaje de desechos orgánicos y criterios de calidad del compost. En: Programa Universitario de Ciencia y Tecnología Agropecuaria (PUI) Ed. Memorias Curso Master Internacional Aprovechamiento de Residuos Orgánicos. Universidad Nacional de Colombia - Sede Palmira, 1994; 1-8.

(8) Ramírez V. Caracterización de las propiedades de diez materiales, prescripción de uso potencial como sustratos y evaluación de crecimiento de plántulas de lechuga. Trabajo de grado. Facultad de Agronomía, Universidad Nacional de Colombia, Bogotá. 2000.

(9) Calderón S, Cavallos F. Los sustratos, (en línea) 2001, (fecha de acceso 15 de marzo de
2016). Disponible en: http://www.drcalderonlabs.com/Publicaciones/Los_Sustratos.htm.

(10) Alarcón A. 2000. Introducción a los cultivos sin suelo. En: Tecnología para cultivos de alto rendimiento. Novedades Agrícolas, España. pp. 191-204.

(11) Alarcón A, Murcia L. Cultivo en fibra de coco. En: Tecnología para cultivos de alto rendimiento. Novedades Agrícolas, España. 2000. pp 245-250.

(11) Fonteno D. Sustratos: tipos y propiedades físicas-quimicas. Reed,D.W.(ed). Agua, sustratos y nutrición en cultivos de flores bajo invernadero. Ediciones Hortitecnia; Ball Publishing, Batavia, IL 1999; 93-123.

(12) Projar. Fibra de coco como sustrato en hidroponía, (en línea) 2016, (fecha de acceso 01 de febrero de 2016). Disponible en: http:// www.interempresas.net/Horticola/Articulos/68605-Fibra-de-coco-como-sustrato-en-hidroponia.html.

(13) Lemaire F. The problem of the biostability in organic substrates. Acta Hortic. 1997; 50: 63-69. DOI: http://dx.doi.org/10.17660/ActaHortic.1997.450.6.

(14) Hartmann H, Kester D, Davies F, Geneve R. Plant propagation: principles and practices. 7a ed. Prentice Hall, Saddle River, NJ. 2002. pp 919.

(15) Cadahía C. Fertirrigación: cultivos hortícolas y ornamentales. 3a ed. Ediciones Mundi-Prensa, Madrid. 2005. pp 681.

(16) Peña M, Casierra F, Monsalve O. Producción hidropónica de tomate (Solanum lycopersicum L.) en cascarilla de arroz mezclada con materiales minerales y orgánicos. Rev. Colomb. Cienc. Hortic, 2013; 7(2): 217-227.

(17) Cabrera R. Propiedades, uso y manejo de sustratos de cultivo para la producción de plantas 
en maceta. Revista Chapingo Serie Horticultura, 1999; 5(1): 5-11.

(18) Pire R, Pereira, A. Propiedades físicas de componentes de sustratos de uso común en la horticultura del estado Lara, Venezuela. Propuesta metodológica. Bioagro, 2003; 15(1): 55-64.

(19) López L, Cárdenas R, Lobit P, Martínez O, Escalante O. Selección de un sustrato para el crecimiento de fresa en hidroponía. Rev. Fitotec. Mex, 2005; 28(2): 171-174.

(20) Pérez de Camacaro M, Ojeda M, Mogollón N, Gimenez, A. Efecto de diferentes sustratos y ácido giberelico sobre el crecimiento, producción y calidad de fresa cv. Camarosa. Bioagro, 2013; 24(1): 31-38

(21) Fraile A, Alvarez J, Deaquiz Y. Efecto de las giberelinas en la propagación de tomate (Solanum lycopersicum L.) bajo diferentes sustratos enriquecidos con fertilizante. Rev. Colomb. Cienc. Hortic, 2012; 6(1): 41-54. DOI: http:// dx.doi.org/10.17584/rcch.2012v6i1.1280.

(22) Jarma A, Buitrago C, Gutiérrez S. Respuesta del crecimiento de la habichuela (Phaseolus vulgaris L. var. Blue Lake) a tres niveles de radiación incidente. Revista Comalfi, 1999; 26(1-3): 62-73.

(23) Hawkins T, Gardiner E, Comer G. Modeling therelationship between extractable chlorophyl land SPAD-502 readings for endangered plant species research. J. Nat. Conserv, 2009; 17: 123-127. DOI: http://dx.doi.org/10.1016/j. jnc.2008.12.007.
(24) Fang L, Feng L, Song, Q, Yuan-S D, Su C, Wang, K. Investigation of SPAD meter-based indices for estimating rice nitrogen status. Computers and Electronics in Agriculture, 2010; 715: 560-565.

(25) Taiz L, Zeiger, E. Plant physiology, 3rd edition. Sinauer Associates Inc. Publishers, Sunderland. 2006; 792 p.

(26) Sampaio R, Ramos S, Guilherme D, Costa C, Fernandes L. Produção de mudas de tomateiro em substratos contendo fibra de coco e pó de rocha. Hortic. Bras, 2008; 26(4): 499503. DOI: http://dx.doi.org/10.1590/S010205362008000400015.

(27) Moreira MA, Dantas FM, Bianchini FG, Viégas PR. Produção de mudas de berinjela com uso de pó de coco. Rev. Bras. Produtos Agroindustriais, 2010; 12(2): 163-170. DOI: http://dx.doi.org/10.15871/1517-8595/rbpa. v12n2p163-170.

(28) Quintero C, Guzmán P, Valenzuela J. 2012. Evaluación de sustratos alternativos para el cultivo de miniclavel (Dianthus caryophyIlus L.). Rev. Colomb. Cienc. Hortic., 6(1): 76-87. DOI: http://dx.doi.org/10.17584/rcch. 2012v6i1.1281.

(29) Adams P. Nutricional control in hydroponics. En: Savvas, D. y H. Passam (eds.). Hydroponic production of vegetables and ornamentals. Embryo Publ., Atenas. 2002; 211-261. 